\title{
The Performance Evaluation of third-party logistics customer service based on Fuzzy Comprehensive Evaluation
}

\author{
Mengyun Xiao ${ }^{1}$, Li Zhou $^{2}$ \\ Beijing Wuzi University, Beijing, China, 101149 \\ (E-mail:xmylogistics@163.com, zhoulibit@126.com)
}

Keywords : Third-party logistics enterprises, KPI system, fuzzy comprehensive evaluation

\begin{abstract}
According to the features of the customer service of third-party logistics, setting up its KPI system of customer service, and then use the fuzzy comprehensive evaluation to evaluate the service performance of third-party logistics enterprises based on the evaluation system, and finally use example to apply the Fuzzy Comprehensive Evaluation Model to the reality of the work of the enterprises, reflecting the usefulness of this method.
\end{abstract}

\section{Introduction}

Nowadays, the market competition has become more and more intense, in order to improve the core competitiveness of enterprises, the non-core business will be outsourced to those professional companies, thus forming the third-party logistics enterprises. In this article, we will use the fuzzy comprehensive evaluation method to evaluate the performance of third-party logistics.

\section{KPI system of service performance for third-party logistics enterprises}

A. KPI System of customer service or third-party logistics enterprises ${ }^{[1]}$

According to services content of third-party logistics, we can divide the KPI system of customer service for third-party logistics into the following three areas: warehousing management, transportation management, financial settlement.

\section{1) Warehousing management}

Through the understanding of all the links in warehouse operations, we can know that the indicator system of warehouse management can be evaluated in four aspects: inventory accuracy, warehousing accuracy, outputting accuracy, cargo damage rate.

\section{2) Transportation management}

This indicator can be defined in six aspects: delivery timeliness, arrival timeliness, customer complaints, customer satisfaction, breakage rate, order fulfillment rate.

\section{3) Financial settlement}

The financial settlement for other companies to evaluate their business service is an important indicator, which includes: settlement timeliness, settlement accuracy.

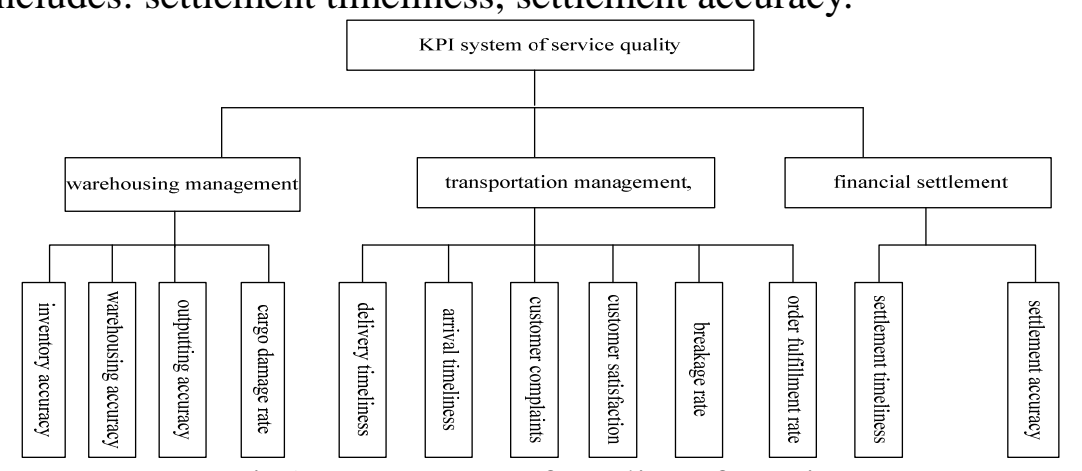

Fig1 KPI system of quality of service 


\section{A. The Definition of Fuzzy Comprehensive Evaluation}

Fuzzy comprehensive evaluation method is a comprehensive evaluation method based on fuzzy mathematics, changing the qualitative evaluation into quantitative evaluation, which uses fuzzy mathematics to make an overall assessment for those objects restricted by many factors. ${ }^{[2]}$.

\section{B. Establish Evaluation Indicator System Set $\mathbf{M}^{[3]}$}

In the service performance system of third-party logistics, we set its evaluation index system as below: $\mathrm{M}=\left(\mathrm{M}_{1}, \mathrm{M}_{2}, \mathrm{M}_{3}\right)$

$\mathrm{M}_{1}$ : warehouse management ; $\mathrm{M}_{2}$ :transportation management ; $\mathrm{M}_{3}$ :financial settlement. And each indicator also contains second-level indicators:

$\mathrm{M}_{1}=\left\{\mathrm{M}_{11}\right.$ (inventory accuracy), $\mathrm{M}_{12}=$ (warehousing accuracy), $\mathrm{M}_{13}=$ (outputting accuracy) , $\mathrm{M}_{14}=$ (cargo damage rate) $\}$;

$\mathrm{M}_{2}=\left\{\mathrm{M}_{21}\right.$ (delivery timeliness), $\mathrm{M}_{22}$ (arrival timeliness), $\mathrm{M}_{23}$ (customer complaints), $\mathrm{M}_{24}$ (customer satisfaction) , $\mathrm{M}_{25}$ (breakage rate), $\mathrm{M}_{26}$ (order fulfillment rate)\};

$\mathrm{M}_{3}=\left\{\mathrm{M}_{31}\right.$ (settlement timeliness), $\mathrm{M}_{32}$ (settlement accuracy) $\}$.

C. Establish Evaluation Set $\mathbf{N}^{[4]}$

It's difficult to quantify the pollution levels, so we need to set up a group of fuzzy evaluation results for each second-level evaluation. For example, we can set up four fuzzy evaluation results for each evaluation: $\mathrm{N}_{1}$ : A (excellent), $\mathrm{N}_{2}$ : $\mathrm{B}$ (good), $\mathrm{N}_{3}$ : C (average), $\mathrm{N}_{4}$ : D (poor).

D. To Determine the Weight Vector of each Indicator

1) The Weight Vector of First-Level Indicators

Calculate the coefficient of the first-level indicators by the score, which makes up the first-level indicator vector, denoted by $U=\left\{U_{1}, U_{2}, \quad, U_{m}\right\}, U_{i}$ is the weight vector of first-level indicator $M_{i}$. $\mathrm{U}_{\mathrm{i}}=\frac{\sum_{\mathrm{j}=1}^{\mathrm{n}} \sum_{\mathrm{s}=1}^{\mathrm{t}} \mathrm{N}_{\mathrm{ijs}}}{\sum_{\mathrm{i}=1}^{\mathrm{m}} \sum_{\mathrm{j}=1}^{\mathrm{n}} \sum_{\mathrm{s}=1}^{\mathrm{t}} \mathrm{N}_{\mathrm{ijs}}}, \quad \mathrm{U}_{\mathrm{i}} \geq, \sum_{\mathrm{j}=1}^{\mathrm{m}} \mathrm{U}_{\mathrm{i}}=1, \mathrm{i}=1,2, \quad, \mathrm{~m}$

2) The Weight Vector of Second-Level Indicators

Similarly, all the coefficients of second-level indicators make up the vector, denoted by $U_{i j}=\left\{U_{i 1}\right.$, $\left.\mathrm{U}_{\mathrm{i} 2}, \quad, \mathrm{U}_{\mathrm{in}}\right\}, \mathrm{U}_{\mathrm{ij}}$ is the weight vector of second-level indicator $\mathrm{M}_{\mathrm{ij}}$.

$\mathrm{U}_{\mathrm{ij}}=\frac{\sum_{\mathrm{s}=1}^{\mathrm{t}} \mathrm{B}_{\mathrm{ijs}}}{\sum_{\mathrm{j}=1}^{\mathrm{n}} \sum_{\mathrm{s}=1}^{\mathrm{t}} B_{\mathrm{ijs}}} \quad, \quad \mathrm{U}_{\mathrm{ij}} \geq 0, \sum_{\mathrm{j}=1}^{\mathrm{m}} \mathrm{U}_{\mathrm{ij}}=1, \mathrm{i}=1,2, \mathrm{n}$

E. Establish Fuzzy Comprehensive Evaluation Matrix ${ }^{[5]}$

After collecting large amount of questionnaires to their long-term business cooperation enterprises, getting the second-level fuzzy evaluation matrix $\mathrm{P}_{\mathrm{k}}$ as follows:

$\mathrm{P}_{\mathrm{k}}=\left[\begin{array}{ccc}\mathrm{a}_{11} & \cdots & \mathrm{a}_{1 \mathrm{~m}} \\ \vdots & \ddots & \vdots \\ \mathrm{a}_{\mathrm{n} 1} & \cdots & \mathrm{a}_{\mathrm{nm}}\end{array}\right] \quad 0 \leq \mathrm{a}_{\mathrm{ij}} \leq 1, \quad \sum_{\mathrm{j}=1}^{\mathrm{m}} \mathrm{a}_{\mathrm{ij}}=1, \mathrm{i}=1,2, \quad, \mathrm{n}$.

$\mathrm{a}_{\mathrm{ij}}=\frac{\text { The number of evalutors selected } \mathrm{j} \text { option for } \mathrm{M}_{\mathrm{ki}}}{\text { The total number of participants }}$

Getting the weight coefficient vector $\mathrm{U}_{\mathrm{ij}}$ of second-level indicators according to the formula, as $\mathrm{N}_{\mathrm{i}}=\mathrm{U}_{\mathrm{ij}} \cdot \mathrm{P}_{\mathrm{k}}$, getting result vector: $\quad \mathrm{N}_{\mathrm{i}}=\left\{\mathrm{b}_{\mathrm{i} 1}, \mathrm{~b}_{\mathrm{i} 2}, \quad, \mathrm{~b}_{\mathrm{in}}\right\}$

The matrix which was consisted of all the second-level result vectors is just the fuzzy evaluation

matrix $\mathrm{P}: \quad \mathrm{P}=\left[\begin{array}{ccc}\mathrm{b}_{11} & \cdots & \mathrm{b}_{1 \mathrm{~m}} \\ \vdots & \ddots & \vdots \\ \mathrm{b}_{\mathrm{n} 1} & \cdots & \mathrm{b}_{\mathrm{nm}}\end{array}\right]$

Similarly, according to the fuzzy transformation formula $\mathrm{N}=\mathrm{M}_{\mathrm{i}} \cdot \mathrm{P}_{\mathrm{i}}$, getting the first-level indicators evaluation results vector $\mathrm{N}$, denoted as $\mathrm{N}=\left\{\mathrm{b}_{1}, \mathrm{~b}_{2}, \quad, \mathrm{~b}_{\mathrm{n}}\right\}$.

\section{Analysis of Numerical examples}

There is a third- party logistics company which wants to conduct an internal assessment of customer service, they conduct a survey for those 200 companies they have long-term cooperation, the findings are as follows: 
Table 1 Weight distribution

\begin{tabular}{|c|c|c|c|}
\hline \multicolumn{2}{|c|}{$1^{\text {st }}$-level indicators } & \multicolumn{2}{|c|}{$2^{\text {nd }}$-level indicators } \\
\hline $\mathrm{M}_{\mathrm{i}}$ & $\mathrm{U}_{\mathrm{i}}$ & $\mathrm{M}_{\mathrm{ij}}$ & $\mathrm{U}_{\mathrm{ij}}$ \\
\hline \multirow[b]{4}{*}{$\mathrm{M}_{1}$} & \multirow[b]{4}{*}{$\mathrm{U}_{1}(0.44)$} & $\mathrm{M}_{11}$ & $\mathrm{U}_{11}(0.22)$ \\
\hline & & $\mathrm{M}_{12}$ & $\mathrm{U}_{12}(0.34)$ \\
\hline & & $\mathrm{M}_{13}$ & $\mathrm{U}_{13}(0.31)$ \\
\hline & & $\mathrm{M}_{14}$ & $\mathrm{U}_{14}(0.13)$ \\
\hline \multirow[b]{6}{*}{$\mathrm{M}_{2}$} & \multirow[b]{6}{*}{$\mathrm{U}_{2}(0.46)$} & $\mathrm{M}_{21}$ & $\mathrm{U}_{21}(0.18)$ \\
\hline & & $\mathrm{M}_{22}$ & $\mathrm{U}_{22}(0.33)$ \\
\hline & & $\mathrm{M}_{23}$ & $U_{23}(0.08)$ \\
\hline & & $\mathrm{M}_{24}$ & $\mathrm{U}_{24}(0.11)$ \\
\hline & & $\mathrm{M}_{25}$ & $\mathrm{U}_{25}(0.14)$ \\
\hline & & $\mathrm{M}_{26}$ & $\mathrm{U}_{26}(0.16)$ \\
\hline \multirow[b]{2}{*}{$\mathrm{M}_{3}$} & \multirow[b]{2}{*}{$\mathrm{U}_{3}(0.10)$} & $\mathrm{M}_{31}$ & $\mathrm{U}_{31}(0.60)$ \\
\hline & & $\mathrm{M}_{32}$ & $\mathrm{U}_{32}(0.40)$ \\
\hline
\end{tabular}

Table 2 Survey distribution

\begin{tabular}{|l|l|l|l|l|}
\hline $\begin{array}{l}\text { indicator } \\
/ 200\end{array}$ & A & B & C & D \\
\hline $\mathrm{M}_{11}$ & 8 & 96 & 96 & 0 \\
\hline $\mathrm{M}_{12}$ & 30 & 122 & 40 & 8 \\
\hline $\mathrm{M}_{13}$ & 11 & 90 & 90 & 9 \\
\hline $\mathrm{M}_{14}$ & 12 & 56 & 104 & 28 \\
\hline $\mathrm{M}_{21}$ & 92 & 80 & 28 & 0 \\
\hline $\mathrm{M}_{22}$ & 96 & 72 & 20 & 12 \\
\hline $\mathrm{M}_{23}$ & 16 & 80 & 84 & 20 \\
\hline $\mathrm{M}_{24}$ & 34 & 92 & 46 & 28 \\
\hline $\mathrm{M}_{25}$ & 32 & 40 & 88 & 40 \\
\hline $\mathrm{M}_{26}$ & 102 & 56 & 30 & 12 \\
\hline $\mathrm{M}_{31}$ & 22 & 42 & 108 & 28 \\
\hline $\mathrm{M}_{32}$ & 46 & 68 & 56 & 30 \\
\hline
\end{tabular}

According to above formula (1) and (2) ,we calculate weight coefficient vector of the first-level and second-level indicators: $\mathrm{M}=(0.44,0.46,0.10), \quad \mathrm{M}_{1}=\quad(0.22,0.34,0.31,0.13$, $\mathrm{M}_{2}=(0.18,0.33,0.08,0.11,0.14,0.16), \mathrm{M}_{3}=(0.60,0.40)$. Also we can calculate each second-level fuzzy evaluation matrix based on the formula (3) and (4) as follows:

$$
\begin{aligned}
& P_{1}=\left[\begin{array}{cccc}
0.04 & 0.48 & 0.48 & 0 \\
0.15 & 0.61 & 0.2 & 0.04 \\
0.05 & 0.45 & 0.45 & 0.05 \\
0.06 & 0.28 & 0.52 & 0.14
\end{array}\right] \\
& P_{3}=\left[\begin{array}{cccc}
0.11 & 0.21 & 0.54 & 0.14 \\
0.23 & 0.34 & 0.28 & 0.15
\end{array}\right]
\end{aligned}
$$$$
\mathrm{P}_{2}=\left[\begin{array}{cccc}
0.46 & 0.40 & 0.14 & 0 \\
0.48 & 0.36 & 0.10 & 0.06 \\
0.08 & 0.40 & 0.42 & 0.10 \\
0.17 & 0.46 & 0.23 & 0.14 \\
0.16 & 0.20 & 0.44 & 0.20 \\
0.51 & 0.28 & 0.15 & 0.06
\end{array}\right]
$$

$\mathrm{N}_{1}=\mathrm{M}_{1} \bullet \mathrm{P}_{1}=(0.22,0.34,0.31,0.13) \cdot\left[\begin{array}{cccc}0.04 & 0.48 & 0.48 & 0 \\ 0.15 & 0.61 & 0.2 & 0.04 \\ 0.05 & 0.45 & 0.45 & 0.05 \\ 0.06 & 0.28 & 0.52 & 0.14\end{array}\right]=(0.0831,0.4889,0.3807,0.0473)$

$\mathrm{N}_{2}=\mathrm{M}_{2} \cdot \mathrm{P}_{2}=(0.18,0.33,0.08,0.11,0.14,0.16) \cdot\left[\begin{array}{cccc}0.46 & 0.40 & 0.14 & 0 \\ 0.48 & 0.36 & 0.10 & 0.06 \\ 0.08 & 0.40 & 0.42 & 0.10 \\ 0.17 & 0.46 & 0.23 & 0.14 \\ 0.16 & 0.20 & 0.44 & 0.20 \\ 0.51 & 0.28 & 0.15 & 0.06\end{array}\right]$

$$
=(0.3703,0.3462,0.2027,0.0809)
$$

$\mathrm{N}_{2}=\mathrm{M}_{2} \cdot \mathrm{P}_{2}=(0.60,0.40) \cdot\left[\begin{array}{cccc}0.11 & 0.21 & 0.54 & 0.14 \\ 0.23 & 0.34 & 0.28 & 0.15\end{array}\right]=(0.158,0.262,0.436,0.144)$

Get the fuzzy evaluation matrix $\mathrm{P}=\left[\begin{array}{l}\mathrm{N}_{1} \\ \mathrm{~N}_{2} \\ \mathrm{~N}_{3}\end{array}\right]$, then $\mathrm{N}=\mathrm{U}_{\mathrm{i}} \cdot \mathrm{P}=\mathrm{M} \cdot \mathrm{P}=(0.44,0.46,0.10) \cdot\left[\begin{array}{l}\mathrm{N}_{1} \\ \mathrm{~N}_{2} \\ \mathrm{~N}_{3}\end{array}\right]$,

$$
=(0.44,0.46,0.10) \cdot\left[\begin{array}{cccc}
0.0831 & 0.4889 & 0.3807 & 0.0473 \\
0.3703 & 0.3462 & 0.2027 & 0.0809 \\
0.158 & 0.262 & 0.436 & 0144
\end{array}\right]=(0.222702,0.400568,0.30435,0.072426)
$$

Normalized handled, $\mathrm{P}=(0.23,0.40,0.30,0.07)$, which shows that the fuzzy comprehensive evaluation results as below: A (excellent):23\%; B (good): 40\%; C (average):30\%; D (poor):7\%. Comprehensive evaluation result is that the third-party logistics service performance is good.

\section{Summary}

With the development of economy, companies have been expanding, so that financial 
management will become increasingly important as an indicator. For a professional third-party logistics, their main business, such as warehousing and transportation has been well developed, in order to further develop, we must strengthen enterprise management, for the management of goods and the training for financial settlement areas is also important.

\section{Acknowledgments}

The paper is supported by the 2011 national statistics research projects "The low-carbon development mode statistical study of the logistics industry" (2011LY115). Supported by Beijing Natural Science Foundation (9133018). Supported by science and technology innovation platform of modern logistics information and control technology research (PXM2012_014214_000067).

\section{References}

[1]Wen Cnen,Qiang Zhang. The grey fuzzy comprehensive evaluation model of customer service performance of third- party logistics [J] Fuzzy Systems and Mathematics, 2007(22):248-253.

[2] Lolo,Mngle Roh,KMne0335,CMNNMge,DMn, Economic analysis/statistical method

[3] Pengfei Zhao,Xiangrui Chen,Yun Zhao. The research on Performance Evaluation of Logistics Enterprises based on the method of fuzzy mathematics [J]. Technique and methods, 2000(330): 305-308.

[4] Guoping Cheng,Shibin Liu.The method and its application of fuzzy comprehensive evaluation of logistics performance based on the service quality[J]. Value Engineering, 2005, 7:57-59.

[5] Ming Yang, Xiaoling Ai,Hongtao Guo,Liye Zhang. Fuzzy Comprehensive Evaluation of the performance of small and medium-sized road freight Logistics [J]. Logistics technology, 2006, 2:45-48 